\title{
Molecule-based osteoarthritis diagnosis comes of age
}

\author{
Zhongyang Lvi, ${ }^{1,2}$ Dongquan Shi ${ }^{1,2}$ \\ ${ }^{1}$ State Key Laboratory of Pharmaceutical Biotechnology, Department of Sports Medicine and Adult Reconstructive Surgery, Nanjing Drum Tower \\ Hospital, The Affiliated Hospital of Nanjing University Medical School, Nanjing, China; ${ }^{2}$ Laboratory for Bone and Joint Disease, Model Animal \\ Research Center (MARC), Nanjing University, Nanjing, China \\ Correspondence to: Prof. Dongquan Shi. State Key Laboratory of Pharmaceutical Biotechnology, Department of Sports Medicine and Adult \\ Reconstructive Surgery, Nanjing Drum Tower Hospital, The Affiliated Hospital of Nanjing University Medical School, 321 Zhongshan Road, \\ Nanjing 210008, China. Email: shidongquan@nju.edu.cn.
}

Submitted Apr 09, 2021. Accepted for publication Jun 24, 2021.

doi: $10.21037 / \mathrm{atm}-21-1745$

View this article at: https://dx.doi.org/10.21037/atm-21-1745

\section{Background}

Osteoarthritis (OA) is a common disabling condition worldwide, representing a substantial and growing health burden with extensive socio-economic costs (1). Patients with OA typically manifest several symptoms that impair their quality of life, such as pain, stiffness, and dysfunction. Even though OA has been recognized as early as the late eighteenth century (2), the current diagnosis of it is largely dependent on clinical information; including symptoms, signs, and images. Such diagnostic criteria are not able to predict high risk OA individuals and/or provide evidence for early diagnosis of the disease. In addition, despite having emerging pharmaceutical therapies in recent decades (3), only a fraction of potentially disease-modifying OA drugs have been applied in clinical practice. Whereas, a portion of these drugs show ambiguous outcomes, and the use of them in clinical guidelines are usually in disagreement $(4,5)$. This could be due to a mismatch between the molecular mechanisms by which the drug works and the clinical manifestations at the time of the decision to use the drug, since the commonly recognized clinical features cannot elucidate the pathological changes of OA (6). Thus, the lack of pathophysiology-based OA diagnosis impedes the development of targeted therapeutics.

\section{OA is a molecular disorder}

$\mathrm{OA}$ is a disease of the whole joint involving the structural changes in the articular cartilage, synovium, subchondral bone, ligaments, and periarticular muscles (1). These structural changes are the cumulative results of changes at the molecular level, as the definition of OA proposed by the Osteoarthritis Research Society International: "The disease manifests first as a molecular derangement (abnormal joint tissue metabolism) followed by anatomic, and/or physiologic derangements (characterized by cartilage degradation, bone remodeling, osteophyte formation, joint inflammation and loss of normal joint function), that can culminate in illness" (7). For instance, under pathological conditions, the depletion of cartilage composition, mainly proteoglycan and collagen, leads to a loss of cartilage integrity and a change in the properties of cartilage material (8). These changes increase the susceptibility to cartilage rupture and eventually result in cartilage degeneration, which can be seen on magnetic resonance imaging (MRI) as thinning of the cartilage or on X-ray as narrowing of the joint space (9) during later stages in OA. Therefore, detection of early altered molecules seems to provide opportunity for a more sensitive and accurate pathological diagnosis of OA.

\section{Transcriptome atlas-based OA diagnosis}

In this context, research in the field of molecular diagnosis of OA has been very active in recent years. Since the pattern of gene expression reflects cell responses to pathological condition, transcriptome data has been used in the diagnosis and classification of OA in several studies. For example, Soul et al. have investigated the pattern of gene expression in non-OA and OA cartilage by RNA-sequencing (RNAseq) (10). To avoid the gene expression alterations that occur in damaged cartilage, they analyzed the intact cartilage in OA group. They showed 2,692 differentially 
expressed genes between non-OA and OA cartilage, and surprisingly, they found a large increase in the expression of matrix protein genes in the OA group. Further unsupervised clustering analysis stratified OA into two subgroups: Group A showed increased expression of cartilage components like collagen type II, V, IX and XI and less expression of collagen type I; in contrast, Group B showed reduced expression of chondrogenic genes and enhanced expression of osteogenic genes. Similarly, Coutinho de Almeida et al. analyzed the whole-transcriptome profiling of OA cartilage and also identified two subgroups (11). Upon integrating radiographic OA data, they found that one subgroup was likely to be characterized by lower osteophyte scores and higher joint space narrowing (JSN) scores. These results clearly show that OA is a highly heterogeneous disease and raises the concept for a more precise molecular diagnosis.

Another example of an unexpected significant discovery was recently published in Bone Research. Different from previous studies that only focused on cartilage, Yuan et al. constructed an extensive transcriptome atlas of $\mathrm{OA}$ cartilage, subchondral bone and synovium, which were mainly affected tissues during OA pathophysiology (12). Through applying the unsupervised clustering analysis, they classified OA into four subtypes: cluster 1 (C1) subtype with glycosaminoglycan metabolic disorder, C2 subtype with collagen metabolic disorder, C3 subtype with activated sensory neuron, and $\mathrm{C} 4$ subtype with inflammation. They further linked the clinical symptoms of different OA subtypes with molecular functions by the ligand-receptor crosstalk analysis of cartilage, subchondral bone and synovium. For example, they found more tissue crosstalk in C4 subtype than others, especially ossification-enriched subchondral bone-cartilage crosstalk and osteoblast differentiation-enriched subchondral bone-subchondral bone crosstalk, suggesting subchondral bone overgrowth and narrowed joint space may be observed in C4. Further, upon clinical data analysis for each OA subtype, they found that $\mathrm{C} 4$ patients had a higher JSN score, demonstrating JSN might be the trait of C4 OA patients. This study is surely of great importance as it links the pathogenesis of OA patients with their clinical manifestations. Their findings provide a new approach for the diagnosis of OA, and the transcriptome atlas may allow for precise diagnosis and targeted therapeutics of $\mathrm{OA}$ in the future.

In recent years, RNA-seq (10-12) and single-cell RNAseq $(13,14)$ have been extensively studied in order to reveal OA subtypes and pathophysiology at molecular and cellular levels. However, it should be critically noted that the transcriptome-based diagnosis has some limitations. First, although transcriptional networks play a fundamental role in governing cell function and fate, they do not entirely determine cellular identity due to ubiquitous posttranscriptional regulation, translational regulation, and degradation mechanisms (15). Confirmation of the link among transcriptome atlas, proteome atlas, and clinical information for OA patients is still pending. Second, the aforementioned transcriptome analysis relies heavily on the technically and invasively acquired joint tissue specimens. It is difficult to generalize in clinical practice, not available for early OA diagnosis and cannot dynamically reflect the pathological changes of the disease, especially for posttreatment evaluation.

\section{Body fluid: readily accessible molecular pool for OA diagnosis}

One of the most important contributors of the OA diagnostic algorithm shifts to a molecule-based assessment is the research on potential biomarkers. According to the World Health Organization definition, a biomarker is "any substance, structure, or process that can be measured in the body or its products and influence or predict the incidence of outcome or disease" (16).

Numerous biomarkers that reflect the pathophysiology of OA can be found in body fluids, including synovial fluid (SF), blood, and urine. SF represents the gold standard fluid for the identification of biomarkers in OA because of its intimate relationship with various joint tissues. Changes in tissue environment will directly affect the SF composition. There is extensive evidence that the level of cartilage oligomeric matrix protein (COMP) in SF is significantly increased after partaking in a marathon (17), suggesting that COMP in SF may be a sensitive indicator of cartilage wear. Blood is easily withdrawn and mediates many of the immunologic pathways. The most widely studied OA biomarkers through blood test are inflammatory cytokines like interlukin- $1 \beta$, tumor necrosis factor- $\alpha$, and C-reactive protein, which are highly associated with symptomatic and radiographic progression of OA $(18,19)$. Their levels may provide the possibility to dynamically monitor the severity and progression of OA. In addition, urine is easily accessible with large volumes and can be acquired with noninvasive techniques. Some metabolites are detectable in urine, like C-telopeptide fragments of Collagen II (CTX-II) and C-telopeptide of Collagen I, which are two recognized indicators of collagen degradation and are associated with 
OA progression $(20,21)$. Of note, the urinary levels of CTXII are responsive to chondroprotective glucosamine (22). In this context, the evaluation of urinary CTX-II levels may allow for the stratification of OA patients who may benefit most from chondroprotective therapeutics and help to monitor the treatment efficacy dynamically.

Taken together, the molecules in body fluids could provide us with lots of information about OA pathogenesis, which will greatly promote the development of molecular diagnosis of OA. By associating molecular profiles in body fluids with clinical information, OA stages and the efficacy of some therapeutics, a more comprehensive and scientific picture should be created to test the clinical application value of molecular diagnosis of OA.

\section{Future perspectives}

In conclusion, the excellent researches mentioned above bring to the light an important issue concerning molecular diagnosis of OA, providing a novel approach for the definition of disease subtypes. Since OA is a highly heterogeneous disease, molecule-based diagnosis raises the possibility that different subgroups may be adapted to different modes of intervention. Therefore, molecular characteristics should be considered in the development of inclusion and exclusion criteria in future clinical trials. Stratifying homogenous patients at the molecular level and selecting therapies targeting their pathogenesis may potentiate the efficacy of some therapeutics. We hope the following studies will focus on deepening the knowledge of molecular profile during $\mathrm{OA}$ initiation and progression, and validating their relevance to clinical practice, which will contribute to further improvements in therapeutic options for patients with OA. In the future, biomarkers in body fluids together with clinical information may become the first screening method for OA diagnosis and classification, and transcriptomic and/or proteomic analysis of joint tissues may be effective tools for validating OA subtypes.

\section{Acknowledgments}

We would like to thank Yannick Xiaofan Yang for his help in polishing our paper.

Funding: None.

\section{Footnote}

Provenance and Peer Review: This article was a standard submission to the journal. The article has undergone external peer review.

Peer Review File: Available at https://dx.doi.org/10.21037/ atm-21-1745

Conflicts of Interest: Both authors have completed the ICMJE uniform disclosure form (available at https:// dx.doi.org/10.21037/atm-21-1745). DS serves as an unpaid Associate Editor-in-Chief of Annals of Translational Medicine from Jun 2019 to May 2024. The other author has no conflicts of interest to declare.

Ethical Statement: The authors are accountable for all aspects of the work in ensuring that questions related to the accuracy or integrity of any part of the work are appropriately investigated and resolved.

Open Access Statement: This is an Open Access article distributed in accordance with the Creative Commons Attribution-NonCommercial-NoDerivs 4.0 International License (CC BY-NC-ND 4.0), which permits the noncommercial replication and distribution of the article with the strict proviso that no changes or edits are made and the original work is properly cited (including links to both the formal publication through the relevant DOI and the license). See: https://creativecommons.org/licenses/by-nc-nd/4.0/.

\section{References}

1. Hunter DJ, Bierma-Zeinstra S. Osteoarthritis. Lancet 2019;393:1745-59.

2. Martel-Pelletier J, Barr AJ, Cicuttini FM, et al. Osteoarthritis. Nat Rev Dis Primers 2016;2:16072.

3. Latourte A, Kloppenburg M, Richette P. Emerging pharmaceutical therapies for osteoarthritis. Nat Rev Rheumatol 2020;16:673-88.

4. Sharma L. Osteoarthritis of the Knee. N Engl J Med 2021;384:51-9.

5. Nguyen C, Lefèvre-Colau MM, Poiraudeau S, et al. Evidence and recommendations for use of intra-articular injections for knee osteoarthritis. Ann Phys Rehabil Med 2016;59:184-9.

6. Kraus VB, Collins JE, Hargrove D, et al. Predictive validity of biochemical biomarkers in knee osteoarthritis: data from the FNIH OA Biomarkers Consortium. Ann Rheum Dis 2017;76:186-95.

7. Kraus VB, Blanco FJ, Englund M, et al. Call for 
standardized definitions of osteoarthritis and risk stratification for clinical trials and clinical use. Osteoarthritis Cartilage 2015;23:1233-41.

8. Loeser RF, Collins JA, Diekman BO. Ageing and the pathogenesis of osteoarthritis. Nat Rev Rheumatol 2016;12:412-20.

9. Katz JN, Arant KR, Loeser RF. Diagnosis and Treatment of Hip and Knee Osteoarthritis: A Review. JAMA 2021;325:568-78.

10. Soul J, Dunn SL, Anand S, et al. Stratification of knee osteoarthritis: two major patient subgroups identified by genome-wide expression analysis of articular cartilage. Ann Rheum Dis 2018;77:423.

11. Coutinho de Almeida R, Mahfouz A, Mei H, et al. Identification and characterization of two consistent osteoarthritis subtypes by transcriptome and clinical data integration. Rheumatology (Oxford) 2021;60:1166-75.

12. Yuan C, Pan Z, Zhao K, et al. Classification of four distinct osteoarthritis subtypes with a knee joint tissue transcriptome atlas. Bone Res 2020;8:38.

13. Chou CH, Jain V, Gibson J, et al. Synovial cell cross-talk with cartilage plays a major role in the pathogenesis of osteoarthritis. Sci Rep 2020;10:10868.

14. Ji Q, Zheng Y, Zhang G, et al. Single-cell RNA-seq analysis reveals the progression of human osteoarthritis. Ann Rheum Dis 2019;78:100-10.

15. Chua BA, Van Der Werf I, Jamieson C, et al. PostTranscriptional Regulation of Homeostatic, Stressed, and

Cite this article as: $\mathrm{Lv}$ Z, Shi D. Molecule-based osteoarthritis diagnosis comes of age. Ann Transl Med 2021;9(14):1112. doi: 10.21037/atm-21-1745
Malignant Stem Cells. Cell Stem Cell 2020;26:138-59.

16. Strimbu K, Tavel JA. What are biomarkers? Curr Opin HIV AIDS 2010;5:463-6.

17. Kim HJ, Lee YH, Kim CK. Biomarkers of muscle and cartilage damage and inflammation during a $200 \mathrm{~km}$ run. Eur J Appl Physiol 2007;99:443-7.

18. Attur M, Belitskaya-Lévy I, Oh C, et al. Increased interleukin- $1 \beta$ gene expression in peripheral blood leukocytes is associated with increased pain and predicts risk for progression of symptomatic knee osteoarthritis. Arthritis Rheum 2011;63:1908-17.

19. Rondanelli M, Braschi V, Gasparri C, et al. Effectiveness of Non-Animal Chondroitin Sulfate Supplementation in the Treatment of Moderate Knee Osteoarthritis in a Group of Overweight Subjects: A Randomized, Double-Blind, Placebo-Controlled Pilot Study. Nutrients 2019;11:2027.

20. Sofat N, Ejindu V, Heron C, et al. Biomarkers in Painful Symptomatic Knee OA Demonstrate That MRI Assessed Joint Damage and Type II Collagen Degradation Products Are Linked to Disease Progression. Front Neurosci 2019;13:1016.

21. Bihlet AR, Byrjalsen I, Bay-Jensen AC, et al. Associations between biomarkers of bone and cartilage turnover, gender, pain categories and radiographic severity in knee osteoarthritis. Arthritis Res Ther 2019;21:203.

22. Nagaoka I, Tsuruta A, Yoshimura M. Chondroprotective action of glucosamine, a chitosan monomer, on the joint health of athletes. Int J Biol Macromol 2019;132:795-800. 